\title{
Neumomediastino espontáneo asociado a episodio grave y persistente de hipo
}

\author{
José L. Patiño-Galeana ${ }^{1 *}$, María P. Reveles-García², Cindy M. Niño-Rodríguez ${ }^{3}$, Karen Suárez-Ramírez e \\ Iris E. Rentería-Solis ${ }^{5}$ \\ ${ }^{1}$ Terapia Respiratoria; ${ }^{2}$ Unidad de Cuidados Intensivos Neonatales; ${ }^{3}$ Radiología Pediátrica; ${ }^{4}$ Residencia de Pediatría; ${ }^{5}$ Otorrinolaringología Pediátrica. \\ Star Medica Infantil Privado, Ciudad de México, México
}

\begin{abstract}
Resumen
Introducción: El neumomediastino espontáneo es la presencia de aire libre en el mediastino que no ocurre por un trauma torácico o esofágico directo. Es un padecimiento muy raro, que usualmente ocurre en pacientes con asma, infecciones respiratorias o aspiración de cuerpo extraño. Se reportan pocos casos asociados a eventos de singultos por reflujo grave. Caso clínico: Se presenta el caso de una paciente de 10 años con neumomediastino espontáneo y extensión al cuello secundario a un episodio grave y persistente de hipo sin antecedentes de asma, trauma, cirugía, infección aguda, aspiración de cuerpo extraño ni ejercicio intenso con Vasalva. Conclusiones: La irritación del tercio distal del esófago produjo los episodios graves y persistentes de hipo que incrementaron la presión intratorácica, que a su vez por efecto Macklin, desencadenó el neumomediastino. El diagnóstico se realiza con estudios de imagen. El tratamiento es conservador, con resolución del cuadro clínico entre 5 y 7 días después de la hospitalización.
\end{abstract}

Palabras clave: Neumomediastino. Enfisema subcutáneo. Hipo. Tomografía. Niños.

\section{Spontaneous pneumomediastinum associated with severe and persistent episode of hiccups}

\begin{abstract}
Background: Spontaneous pneumomediastinum is the presence of free air in the mediastinum that does not occur from direct thoracic or esophageal trauma. It is a very rare condition usually related to patients with asthma, respiratory infections, or foreign body aspiration. Only a few cases are reported to be associated with severe reflux events. Case report: We present the case of a 10-year-old female with spontaneous pneumomediastinum extending to the neck secondary to severe and persistent hiccups, with no history of asthma, trauma, surgery, acute infection, foreign body aspiration, and intense exercise with Vasalva. Conclusions: The irritation of the distal third of the esophagus produced severe and persistent episodes of hiccups that increased the intrathoracic pressure, which in turn triggered the pneumomediastinum by Macklin effect. The diagnosis is made with imaging studies. The treatment is conservative with resolution of the clinical picture between five and seven days after hospitalization.
\end{abstract}

Keywords: Pneumomediastinum. Subcutaneous emphysema. Hiccups. Tomography. Children.

\section{Correspondencia:}

*José Luis Patiño Galeana

E-mail: Jlpatino.hip@starmedica.com
Fecha de recepción: 22-10-2020

Fecha de aceptación: 02-02-2021 DOI: 10.24875/BMHIM.20000332
Disponible en internet: 17-09-2021 Bol Med Hosp Infant Mex. 2021;78(5):485-488

www.bmhim.com 1665-1146/@ 2021 Hospital Infantil de México Federico Gómez. Publicado por Permanyer. Este es un artículo open access bajo la licencia CC BY-NC-ND (http://creativecommons.org/licenses/by-nc-nd/4.0/). 


\section{Introducción}

El neumomediastino espontáneo con extensión al cuello es una afección rara. El $0.3 \%$ de los niños que ingresan a urgencias con una crisis asmática presentan neumomediastino como complicación ${ }^{1}$. El primer caso de neumomediastino espontáneo fue descrito por Hamman en 1939. Habitualmente se produce por la rotura alveolar secundaria al incremento de la presión intratorácica por maniobras de Valsalva.

Existen pocos casos descritos de neumomediastino asociados con eventos de hipo ${ }^{2}$. El hipo es un reflejo involuntario que inicia con la contracción del diafragma y termina abruptamente con el cierre de la glotis ${ }^{3}$. Las manifestaciones más comunes en adolescentes son dolor torácico, dolor cervical y disfagia. El diagnóstico se establece por estudios de radiología y el tratamiento es conservador en la mayoría de las ocasiones ${ }^{4}$. El presente caso de neumomediastino espontáneo fue causado por un evento grave y persistente de hipo en una paciente con reflujo.

\section{Caso clínico}

Se presenta el caso de una niña de 10 años con antecedente de dolor abdominal y sensación de pirosis de 1 mes de evolución. Acudió al servicio de urgencias por presentar dolor torácico súbito y opresivo en la región retroesternal, disnea leve, disfagia a líquidos y dolor a la movilización de la región cervical. Cuatro horas antes había presentado dos eventos graves y persistentes de hipo, de 30 y 40 minutos de duración cada uno. Ingresó con los siguientes signos vitales: frecuencia cardiaca 70 latidos/min, frecuencia respiratoria $24 \mathrm{resp} / \mathrm{min}$, temperatura $36.2{ }^{\circ} \mathrm{C}$, saturación de oxígeno $96 \%$ y presión arterial 115/53 mmHg. En la exploración física se encontró despierta, consciente, con adecuada coloración de tegumentos, sin facies de dolor, pupilas isocóricas normorreflécticas, narinas, otoscopia y faringe sin alteraciones, cuello con dolor a la palpación y movilización cervical bilateral, no se palpó enfisema, no había ingurgitación yugular, tórax sin datos de dificultad respiratoria, murmullo vesicular ligeramente disminuido en la base derecha, vibraciones vocales normales, transmisión bilateral de la voz, sin adventicios, tórax anterior sin soplos, crepitación ni enfisema, extremidades con llenado capilar de $2 \mathrm{~s}$, y pulsos simétricos bilaterales sin modificación con la inspiración ni la espiración.

Los resultados de la biometría hemática fueron los siguientes: hemoglobina $14.0 \mathrm{~g} / \mathrm{dll}$, hematocrito $42.3 \%$,

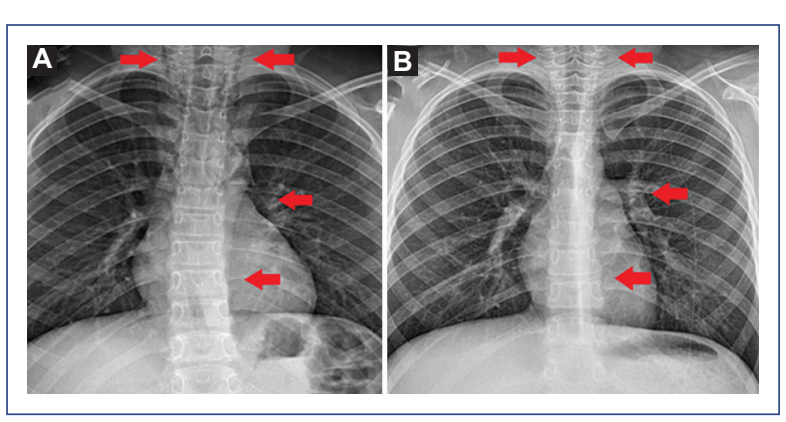

Figura 1. A: radiografía simple de tórax en proyección posteroanterior al ingreso. Se visualiza una mayor amplitud del ancho mediastinal por líneas radiotransparentes que se proyectan en los tejidos blandos mediastinales desde la región cervical (flechas superiores) hasta el borde cardiaco de ambos lados (flecha del centro), que incluso delimitan la línea paravertebral inferior izquierda (flecha inferior), sugestivas de aire libre en el mediastino. B: control 5 días después del ingreso hospitalario. La radiografía simple de tórax en proyección posteroanterior muestra una menor amplitud del ancho mediastinal (flechas superiores), con mejor definición de las líneas grasas paratraqueales (flecha del centro), las líneas paravertebrales (flecha inferior) y los tejidos blandos del cuello, donde se observa la resolución completa del aire mediastinal.

volumen corpuscular medio $90 \mathrm{fl}$, hemoglobina corpuscular media 29.7 pg, concentración de hemoglobina corpuscular media $33.2 \mathrm{~g} / \mathrm{dl}$, leucocitos $13.2 \times 10^{3} / \mu \mathrm{l}$, neutrófilos $72 \%\left(9.5 \times 10^{3} / \mu \mathrm{l}\right)$, linfocitos $22 \%(2.90 \times$ $\left.10^{3} / \mu \mathrm{l}\right)$, monocitos $6 \%\left(0.79 \times 10^{3} / \mu \mathrm{l}\right)$, eosinófilos $0 \%$, bandas $0 \%$, plaquetas $285 \times 10^{3} / \mu$ y proteína $C$ reactiva $0.31 \mathrm{mg} / \mathrm{dl}$.

Se realizaron radiografías lateral y anteroposterior de cuello, y posteroanterior de tórax (Figura 1 A). Por los datos clínicos sugestivos de neumomediastino a tensión (dolor torácico, disnea y dolor a la palpación y a la movilización cervical) se decidió realizar una tomografía computarizada (TC) simple de cuello y tórax para valorar la extensión de la lesión (Figura 2 A). También se realizó un esofagograma para descartar rotura esofágica (Figura $3 \mathrm{~A}$ ).

Se pautó oxígeno con bolsa mascarilla reservorio a $10 \mathrm{l} / \mathrm{min}$, ayuno, paracetamol (15 mg/kg/dosis), omeprazol ( $1 \mathrm{mg} / \mathrm{kg} / \mathrm{día})$ y esteroide inhalado. Ingresó a la unidad de terapia intermedia pediátrica para vigilancia y monitorización continua, ya que en el servicio de urgencias presentó progresión de la disnea de leve a grave.

Permaneció en terapia intermedia por 72 horas, con evolución favorable. Se realizó una TC de cuello y tórax 


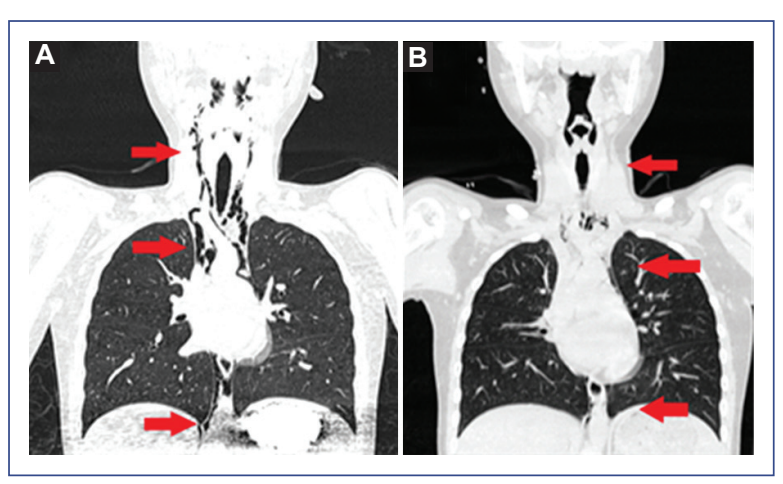

Figura 2. A: tomografía computarizada simple de tórax en plano coronal y ventana para pulmón. Se identifica una moderada cantidad de aire que diseca los planos profundos cervicales, la grasa de los vasos supraaórticos, paracardiaca y de la región paraesofágica inferior, lo que confirma el neumomediastino. B: control tomográfico 3 días después del ingreso hospitalario. Se observa la resolución casi completa del neumomediastino en el plano coronal de la ventana para pulmón, con aire laminar residual.

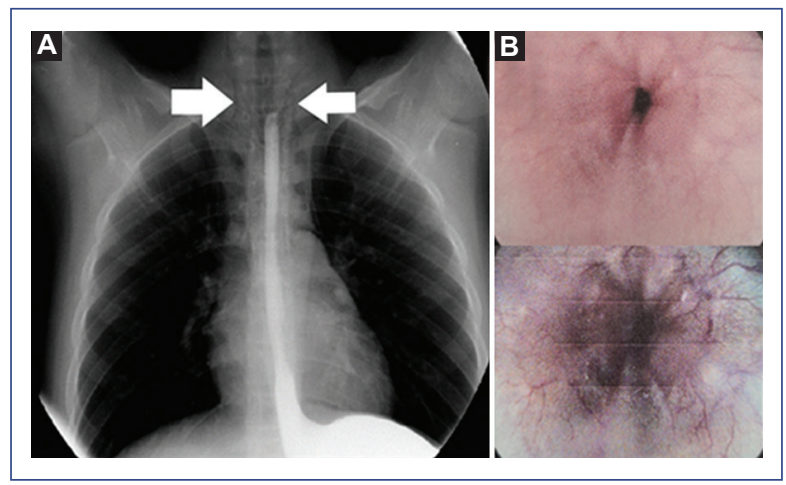

Figura 3. A: serie esofagogastroduodenal que muestra la devolución del contraste baritado de la cavidad abdominal hacia el tercio superior del esófago, sin evidencia de fugas de contraste ni comunicaciones con el neumomediastino (flechas). B: estudio endoscópico de la vía digestiva en el cual se observa el tercio distal del esófago con mucosa hiperémica y erosiones menores de $5 \mathrm{~mm}$.

a las 72 horas, en la que se observó una disminución progresiva de la sintomatología cervical y torácica (Figura 2 B). También se realizó otra endoscopia de la vía digestiva y la vía aérea ante la persistencia del dolor abdominal y pirosis, y se observó esofagitis de grado $\mathrm{A}$ de Los Ángeles (Figura 3 B). Histopatológicamente se reportó esofagitis con actividad moderada, gastritis con actividad moderada y duodenitis leve con hiperplasia linfoide. En el lavado broncoalveolar se halló una elevada cantidad de macrófagos alveolares cargados de lípidos sugestivos de aspiración (índice de lipófagos: 120).

A los 6 días se realizó otra radiografía de cuello y tórax que mostró la resolución total del neumomediastino (Figura 1 B). La paciente egresó ventilatoria y hemodinámicamente estable.

\section{Discusión}

El neumomediastino espontáneo es muy raro en pacientes pediátricos. En el servicio de urgencias se reporta una incidencia de hasta 1/44,000 pacientes. Es más común en el sexo masculino y la edad media de presentación oscila entre los 7 y los 14 años. Habitualmente se asocia con padecimientos respiratorios crónicos, como asma, pero también puede ser causado por un incremento de la presión intratorácica por otros padecimientos agudos, como la aspiración de un cuerpo extraño y las infecciones de vías respiratorias bajas $^{4-6}$. Se han reportado muy pocos casos de neumomediastino secundario a eventos de hipo en adolescentes. En el caso aquí descrito se estableció que los receptores periféricos del nervio vago se estimularon por la presencia de reflujo gastroesofágico en el tercio distal del esófago secundario a una irritación causada por la ingesta rápida de alimentos condimentados. Esto propició los episodios graves y persistentes de hipo con una duración de varios minutos ${ }^{7}$, señal de que el estímulo viajó directamente al sistema nervioso central, provocando la contracción abrupta e involuntaria del diafragma que, a su vez, incrementó la presión intratorácica y produjo la rotura alveolar y la disección de la vaina peribroncovascular, con diseminación del enfisema intersticial pulmonar hacia el mediastino (efecto Macklin), y se extendió por las fascias del cuello hasta el espacio retrofaríngeo y el espacio paratraqueal ${ }^{8,9}$.

La sintomatología más común consiste en dolor torácico, dolor cervical y disfagia, como en este caso. Otros síntomas que pueden presentar los pacientes con neumomediastino espontáneo son disnea, dolor facial, dolor a la palpación en el cuello, estridor, odinofagia y ansiedad ${ }^{10,11}$. A pesar de tratarse de una condición benigna, ya que no se llega a requerir ventilación mecánica ni procedimientos quirúrgicos, se describe que hasta el $20 \%$ de los pacientes han ingresado a cuidados intensivos por el riesgo de desarrollar complicaciones, como neumotórax y neumomediastino a tensión, neumopericardio y mediastinitis ${ }^{12}$.

El diagnóstico se establece por estudios de imagen. En un reporte de 87 casos con neumomediastino espontáneo, Wong, et al. $^{13}$ demostraron que la 
radiografía de tórax presentaba el $89 \%$ de sensibilidad, frente al $95 \%$ de la radiografía lateral de cuello. Sin embargo, existen reportes de sensibilidad del $100 \%$ con ambas radiografías ${ }^{10}$. Se sugiere realizar una TC de cuello y tórax para valorar la extensión de la lesión y descartar una compresión extrínseca que comprometa los grandes vasos por un neumomediastino a tensión ${ }^{14}$. Adicionalmente, se puede realizar un esofagograma para descartar una rotura esofágica (síndrome de Boerhaave $)^{15}$, y una endoscopia de la vía digestiva y aérea como parte del algoritmo terapéutico para establecer con certeza la causa del neumomediastino ${ }^{9}$.

En diversos reportes, el tratamiento del neumomediastino incluye oxigenoterapia, vigilancia del patrón respiratorio y analgésicos ${ }^{16,17}$. En los pacientes asmáticos se recomienda el uso de broncodilatadores de acción corta y esteroides inhalados ${ }^{18}$. En la mayoría de los pacientes, con tratamiento conservador, la resolución se produce en un periodo de 2 a 12 días $^{19}$.

En este reporte se aborda el caso de una paciente con neumomediastino espontáneo por hipo (sumamente infrecuente en pediatría), por lo que la presentación clínica es de interés para facilitar su reconocimiento y conocer su tratamiento.

\section{Responsabilidades éticas}

Protección de personas y animales. Los autores declaran que para esta investigación no se han realizado experimentos en seres humanos ni en animales.

Confidencialidad de los datos. Los autores declaran que han seguido los protocolos de su centro de trabajo sobre la publicación de datos de pacientes.

Derecho a la privacidad y consentimiento informado. Los autores han obtenido el consentimiento informado de los pacientes 0 individuos referidos en el artículo. Este documento obra en poder del autor de correspondencia.

\section{Conflicto de intereses}

Los autores declaran no tener ningún conflicto de intereses.

\section{Financiamiento}

Ninguno.

\section{Agradecimientos}

A todos los autores, por haber colaborado de manera integral con una visión pediátrica y particularizada para la elaboración del caso clínico.

\section{Bibliografía}

1. Takada K, Matsumoto S, Hiramatsu T, Kojima E, Shizu M, Okachi S, et al. Spontaneous pneumomediastinum: an algorithm for diagnosis and management. Ther Adv Respir Dis. 2009;3:301-7.

2. Na SJ, Lee SI, Chung TS, Choi YC, Lee KY. Pneumomediastinum due to intractable hiccup as the presenting symptom of multiple sclerosis. Yonsei Med J. 2005;46:292-5.

3. Yallanki N, Wilks-Gallo L, Cifuni JL, Small-Harary L. Case 3: persistent hiccups and vomiting in an adolescent. Pediatr Rev. 2020;41:423-6.

4. Gasser CR, Pellaton R, Rochat CP. Pediatric spontaneous pneumomediastinum: narrative literature review. Pediatr Emerg Care. 2017;33:370-4

5. López EM, Rivera AQ. Neumomediastino y neumotórax recurrente. Pediatr Mex. 2011;13:155-9.

6. Tortajada-Girbés M, Moreno-Prat M, Ainsa-Laguna D, Mas S. Spontaneous pneumomediastinum and subcutaneous emphysema as a complication of asthma in children: case report and literature review. Ther Adv Respir Dis. 2016;10:402-9.

7. Steger M, Schneemann M, Fox M. Systemic review: the pathogenesis and pharmacological treatment of hiccups. Aliment Pharmacol Ther. 2015;42:1037-50.

8. Wintermark M, Schnyder P. The Macklin effect: a frequent etiology for pneumomediastinum in severe blunt chest trauma. Chest. 2001;120:543-7.

9. Cho DY, Aaron GP, Shepard KG. Spontaneous retropharyngeal and mediastinal emphysema. Clin Exp Otorhinolaryngol. 2016;9:178-81.

10. Kim SH, Huh J, Song JY, Kang IS. Spontaneous pneumomediastinum: a rare disease associated with chest pain in adolescents. Yonsei Med J. 2015;56:1437-42.

11. Kira K, Inokuchi R, Maehara H, Tagami S. Spontaneous pneumomediastinum. BMJ Case Rep. 2016;2016:988-91.

12. Fitzwater JW, Silva NN, Knight CG, Malvezzi L, Ramos-Irizarry C, Burnweit CA. Management of spontaneous pneumomediastinum in children. J Pediatr Surg. 2015;50:983-6.

13. Wong KS, Wu HM, Lai SH, Chiu CY. Spontaneous pneumomediastinum: analysis of 87 pediatric patients. Pediatr Emerg Care. 2013;29:988-91.

14. Cheong L, Koh ES, Teo DB. Pay Air-Tension: anorexia and tension pneumomediastinum. Am J Med. 2019;132:1292-4.

15. Antonis $\mathrm{JH}$, Poeze M, Van Heurn LW. Boerhaave's syndrome in children: a case report and review of the literature. J Pediatr Surg. 2006;41:1620-3.

16. Chidambaram A, Donekal S. Spontaneous pneumomediastinum and subcutaneous emphysema in a child with unknown aetiology. BMJ Case Rep. 2019;12:2018-20.

17. Ebina M, Inoue A, Takaba A, Ariyoshi K. Management of spontaneous pneumomediastinum: are hospitalization and prophylactic antibiotics needed? Am J Emerg Med. 2017;35:1150-3.

18. Damore DT, Dayan PS. Medical causes of pneumomediastinum in children. Clin Pediatr (Phila). 2001;40:87-91.

19. Bullaro FM, Bartoletti SC. Spontaneous pneumomediastinum in children: a literature review. Pediatr Emerg Care. 2007;23:28-30. 